\title{
Carbon Nanotube Based Composites- A Review
}

\author{
Rupesh Khare*, Suryasarathi Bose \\ Mumbai University Institute of Chemical Technology (UICT) \\ rupesh_uict@yahoo.com \\ *author to whom all the correspondence should be addressed
}

\begin{abstract}
:
Carbon nanofibers and nanotubes are promising to revolutionise several fields in material science and are a major component of nanotechnology. Further market development will depend on material availability at reasonable prices. Nanotubes have a wide range of unexplored potential applications in various technological areas such as aerospace, energy, automobile, medicine, or chemical industry, in which they can be used as gas adsorbents, templates, actuators, composite reinforcements, catalyst supports, probes, chemical sensors, nanopipes, nano-reactors etc. In this paper, recent research on carbon nanotube composites are reviewed. The interfacial bonding properties, mechanical performance, electrical percolation of nanotube/polymer and ceramic are also reviewed.
\end{abstract}

\section{INTRODUCTION}

\section{Carbon nanotube}

Elemental carbon in the $\mathrm{sp}^{2}$ hybridization can form a variety ${ }^{1}$ of amazing structures. Apart from the well-known graphite, carbon can build closed and open cages with honeycomb atomic arrangement. The first such structure to be discovered was the C60 molecule by $\mathrm{Kroto}^{2}$ et al. Although various carbon cages were studied, it was only in 1991, when Iijima ${ }^{3}$ observed for the first time tubular carbon structures. The nanotubes consisted of up to several tens of graphitic shells (socalled multi-walled carbon nanotubes (MWNT)) with adjacent shell separation of $\sim 0.34$

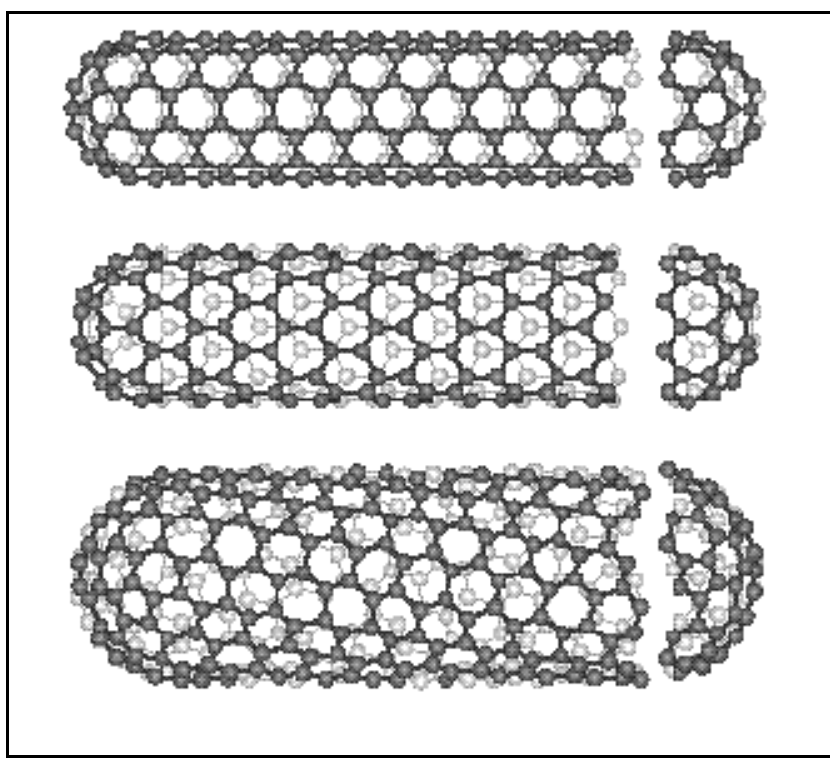

Figure 1: Single wall carbon nanotubes $\mathrm{nm}$, diameters of $\sim 1 \mathrm{~nm}$ and high length/diameter ratio. Two years later, Iijima and Ichihashi $^{4}$ and Bethune et al. ${ }^{5}$ synthesized single-walled carbon nanotubes (SWNT) (figure1). 
There are two main types of carbon nanotubes ${ }^{6}$ that can have high structural perfection. Singlewalled nanotubes (SWNT) consist of a single graphite sheet seamlessly wrapped into a cylindrical tube. Multiwalled nanotubes (MWNT) comprise an array of such nanotubes that are concentrically nested like rings of a tree trunk (figure 2).

\section{Synthesis of $C N$}

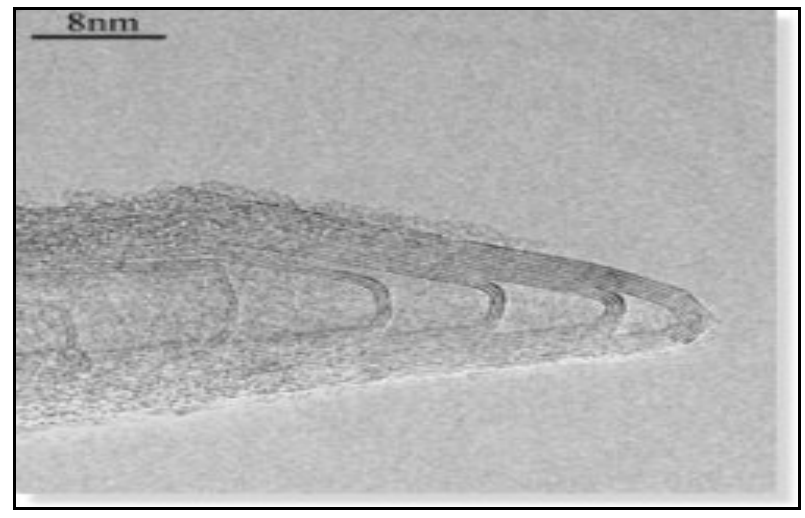

Figure 2: tip of multi wall carbon nanotube

The MWNT were first discovered ${ }^{1}$ in the soot of the arc-discharge method by Iijima. This method had been used long before in the production of carbon fibers and fullerenes. It took two more years for Iijima and Ichihashi ${ }^{4}$, and Bethune ${ }^{5}$ et al. to synthesize SWNT by use of metal catalysts in the arc-discharge method in 1993. Significant progress was achieved by laser-ablation synthesis of bundles of aligned SWNT with small diameter distribution by Smalley and co-workers ${ }^{7}$. Catalytic growth of nanotubes by the chemical vapor decomposition (CVD) method was first used by Yacaman ${ }^{8}$ et al.

\section{Arc-discharge}

In 1991, Iijima reported the preparation of a new type of finite ${ }^{1}$ carbon structures consisting of needle-like tubes ${ }^{3}$. The tubes were produced using an arcdischarge evaporation method similar to that used for the fullerene synthesis. The carbon needles, ranging from 4 to $30 \mathrm{~nm}$ in diameter and up to $1 \mathrm{~mm}$ in length, were grown on the negative end of the carbon electrode used for the direct current (dc) arc-discharge evaporation of carbon in an argon-filled vessel (100 Torr) (see figure 3). Ebbesen and Ajayan $^{9}$ reported large-scale synthesis of MWNT by a variant of the standard arcdischarge technique. Iijima used an arcdischarge chamber filled with a gas mixture

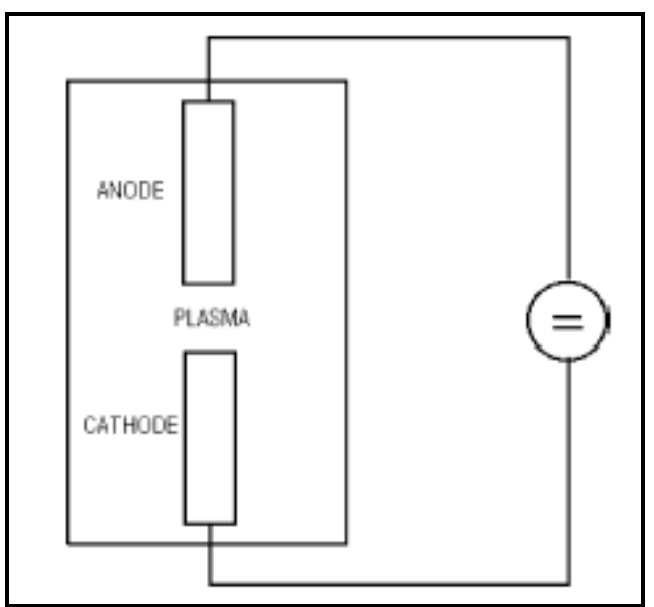

Figure 3: Arc-discharge scheme. Two graphite electrodes are used to produce a dc electric arc-discharge in inert gas atmosphere. of 10 Torr methane and 40 Torr argon. Two vertical thin electrodes were installed in the center of the chamber. The lower electrode, the cathode, had a shallow dip to hold a small piece of iron during the evaporation. The arc-discharge was generated by running a dc current of $200 \mathrm{~A}$ at $20 \mathrm{~V}$ between the 
electrodes. The use of the three components-argon, iron and methane, was critical for the synthesis of SWNT. The nanotubes had diameters of $1 \mathrm{~nm}$ with a broad diameter distribution between 0.7 and $1.65 \mathrm{~nm}$. In the arc-discharge synthesis of nanotubes, Bethune ${ }^{5}$ et al. used as anodes thin electrodes with bored holes, which were filled with a mixture of pure powdered metals $(\mathrm{Fe}, \mathrm{Ni}$ or $\mathrm{Co})$ and graphite. The electrodes were vaporized with a current of 95-105 A in 100-500 Torr of He. Large quantities of SWNT were generated by the arc-technique by Journet ${ }^{10}$ et al. The arc was generated between two graphite electrodes in a reactor under helium atmosphere (660 mbar).

\section{Laser-ablation}

In 1996, Smalley and coworkers produced high yields $(>70 \%)$ of SWNT by laserablation (vaporization) of graphite rods with small amounts ${ }^{7}$ of $\mathrm{Ni}$ and $\mathrm{Co}$ at $1200{ }^{\circ} \mathrm{C}$ (see figure 4). The tube grows until too many catalyst atoms aggregate on the end of the nanotube. The large particles either detach or become over-coated with sufficient carbon to poison the catalysis. This allows the tube to terminate with a fullerene-like tip or with a catalyst particle. Both arc-discharge and

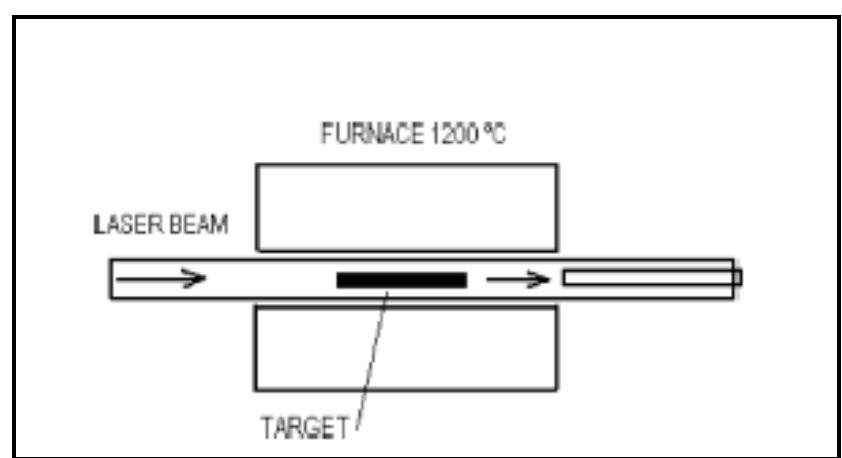

Figure 4:Laser-ablation scheme: Laser beam vaporizes target of a mixture of graphite and metal catalyst (Co, $\mathrm{Ni}$ ) in a horizontal tube in a flow of inert gas at controlled pressure and in a tube furnace at $1200{ }^{\circ} \mathrm{C}$. The nanotubes are deposited on a water-cooled collector outside the furnace laser-ablation techniques have the advantage of high $(>70 \%)$ yields of SWNT and the drawback that (1) they rely on evaporation of carbon atoms from solid targets at temperatures $>3000{ }^{\circ} \mathrm{C}$, and (2) the nanotubes are tangled which makes difficult the purification and application of the samples.

\section{Chemical vapour deposition (CVD)}

Despite the described progress of synthetic techniques ${ }^{12}$ for nanotubes, there still remained two major problems in their synthesis, i.e. large scale production and ordered synthesis. But, in 1996 a CVD method emerged as a new candidate for nanotube synthesis. This method is capable of controlling growth direction on a substrate and synthesising ${ }^{13}$ a large quantity of nanotubes. In this process a mixture of hydrocarbon gas, acetylene, methane or ethylene and nitrogen was introduced into the reaction chamber. During the reaction, nanotubes were formed on the substrate by the decomposition of the hydrocarbon at temperatures $700-900^{\circ} \mathrm{C}$ and atmospheric ${ }^{14}$ pressure The process has two main advantages: the nanotubes are obtained at much lower 
temperature, although this is at the cost of lower quality, and the catalyst can be deposited on a substrate, which allows for the formation of novel structures.

\section{The substrate}

The preparation of the substrate and the use of the catalyst deserve special attention, because they determine the structure of the tubes. The substrate is usually silicon, but also, glass and alumina are used. The catalysts are metal nanoparticles, like $\mathrm{Fe}$, Co and Ni, which can be deposited on silicon substrates either from solution, electron beam evaporation or by physical sputtering. The nanotube diameter depends on the catalyst particle size, therefore, the catalyst deposition technique, in particular the ability to control the particle size, is critical to develop nanodevices. Porous silicon is an ideal substrate for growing self-oriented nanotubes on large surfaces. It has been proven that nanotubes grow at a higher ratio (length per minute), and they are better aligned than on plain silicon ${ }^{15}$. The nanotubes grow parallel to each other and perpendicular to the substrate surface, because of catalyst-surface interaction and the van der Waals forces developed between the tubes.

\section{The sol-gel}

The sol-gel method uses a dried silicon gel, which has undergone several chemical processes, to grow highly aligned nanotubes. The substrate can be re-used after depositing new catalyst particles on the surface. The length of the nanotube arrays increases with the growth time, and reaches about $2 \mathrm{~mm}$ after 48 -h growth ${ }^{14}$.

\section{Gas phase metal catalyst}

In the methods described above, the metal catalysts are deposited or embedded on the substrate before the deposition of the carbon begins. A new method is to use a gas phase for introducing the catalyst, in which both the catalyst and the hydrocarbon gas are fed into a furnace, followed by catalytic reaction in the gas phase. The latter method is suitable for large-scale synthesis, because the nanotubes are free from catalytic supports and the reaction can be operated continuously. A high-pressure carbon monoxide (CO) reaction method, in which $\mathrm{CO}$ gas reacts with iron pentacarbonyl, $\mathrm{Fe}(\mathrm{CO})_{5}$ to form SWNT, has been developed ${ }^{7}$. SWNT have also been synthesized from a mixture of benzene and ferrocene, $\mathrm{Fe}\left(\mathrm{C}_{5} \mathrm{H}_{5}\right)_{2}$ in a hydrogen gas flow ${ }^{17}$. In both methods, catalyst nanoparticles are formed through thermal decomposition of organo metallic compounds, such as iron pentacarbonyl and ferrocene. 
The reverse micelle method is promising, which contains catalyst nanoparticles (Mo and Co) with a relatively homogeneous size distribution in a solution. The presence of surfactant makes the nanoparticles soluble in an organic solvent, such as toluene and benzene. The colloidal solution can be sprayed into a furnace, at a temperature of $1200^{\circ} \mathrm{C}$; it vaporizes simultaneously with the injection and a reaction occurs to form a carbon product. The toluene vapour and metal nanoparticles act as carbon source and catalyst, respectively. The carbon product is removed from the hot zone of the furnace by a gas stream (hydrogen) and collected at the bottom of the chamber ${ }^{18}$.

\section{Recent trends in the synthesis of CNT}

Kirsten Edgar and John L. Spencer ${ }^{21}$ synthesized carbon nanotubes from an aerosol precursor. Solutions of transition metal cluster compounds were atomized by electro hydrodynamic means and the resultant aerosol was reacted with ethyne in the gas phase to catalyse the formation of carbon nanotubes. The use of an aerosol of iron pentacarbonyl resulted in the formation of multi-walled nanotubes, mostly 6-9 nm in diameter, whereas the use of iron dodecacarbonyl gave results that were concentration dependent. High concentrations resulted in a wide diameter range $(30-200 \mathrm{~nm})$ whereas lower concentrations gave multi-walled nanotubes with diameters of 19-23 nm.

Luciano Andrey Montoro et al synthesized ${ }^{22}$ SWNT by arc water process. They could synthesize high-quality SWNT and MWNT through arc-discharge in $\mathrm{H}_{3} \mathrm{VO}_{4}$ aqueous solution from pure graphite electrodes. They suggested that the VO group acts as a nucleation agent promoting the growth of this more ordered carbon structure. The $\mathrm{H}_{3} \mathrm{VO}_{4}$ compound was used to avoid the presence of metallic cations, and it was obtained from a reaction commonly used for the synthesis of xerogels ${ }^{23}$.

Jieshan et al. prepared ${ }^{24}$ CNT by electrically arcing carbon rods in helium (99.99\%) in a stainless steel chamber with an inner diameter of $600 \mathrm{~mm}$ and a height of $350 \mathrm{~mm}$. The anode was a coal-derived carbon rod $(10 \mathrm{~mm}$ in diameter, $100-200 \mathrm{~mm}$ in length); the cathode was a high-purity graphite electrode (16 $\mathrm{mm}$ in diameter, $30 \mathrm{~mm}$ in length). The helium gas functioned as buffer gas and its pressure was varied in range of 0.033-0.076 MPa in the experiment. The voltage and current for arcing were controlled at 30-40 $\mathrm{V}$ and 50-70 A, respectively.

Mingwang Shao ${ }^{25}$ et al. synthesized CNT via a novel route using an iron catalyst at the extremely low temperature of $180^{\circ} \mathrm{C}$. In this process, carbon sub oxide was used as carbon source, which changed to freshly formed free carbon clusters through disproportionation. The carbon clusters can grow into nanotubes in the presence of $\mathrm{Fe}$ catalyst, which was obtained by the decomposition of iron carbonyl $\mathrm{Fe}_{2}(\mathrm{CO})_{9}$ at $250{ }^{\circ} \mathrm{C}$ under nitrogen atmosphere.

SWNT have been successfully synthesized using a fluidized-bed method ${ }^{26}$ that involves fluidization of a catalyst/support at high temperatures by a hydrocarbon flow, 
shows promise for the large-scale, potentially continuous, production of SWNT, at high yield.

A new method, which combines non-equilibrium plasma reaction ${ }^{27}$ with templatecontrolled growth technology, has been developed for synthesizing aligned carbon nanotubes at atmospheric pressure and low temperature. Multiwall carbon nanotubes with diameters of approximately $40 \mathrm{~nm}$ were restrictedly synthesized in the channels of anodic aluminum oxide template from a methane hydrogen mixture gas by a.c. corona discharge plasma reaction at a temperature below $200{ }^{\circ} \mathrm{C}$.

In a recent technique, Nebulized spray pyrolysis ${ }^{28}$, large-scale synthesis of MWNT and aligned MWNT bundles is reported. Nebulized spray is a spray generated by an ultrasonic atomizer. MWNT with fairly uniform diameters as well as aligned MWNT bundles have been obtained by using solutions of organometallic compounds such as ferrocene in benzene, toluene and other hydrocarbon solvents. A SEM image of aligned MWNT bundles obtained by the pyrolysis of a nebulized spray of ferrocene-tolueneacetylene mixture .The advantage of using a nebulized spray is the ease of scaling into an industrial scale process, as the reactants are fed into the furnace continuously (figure 6).

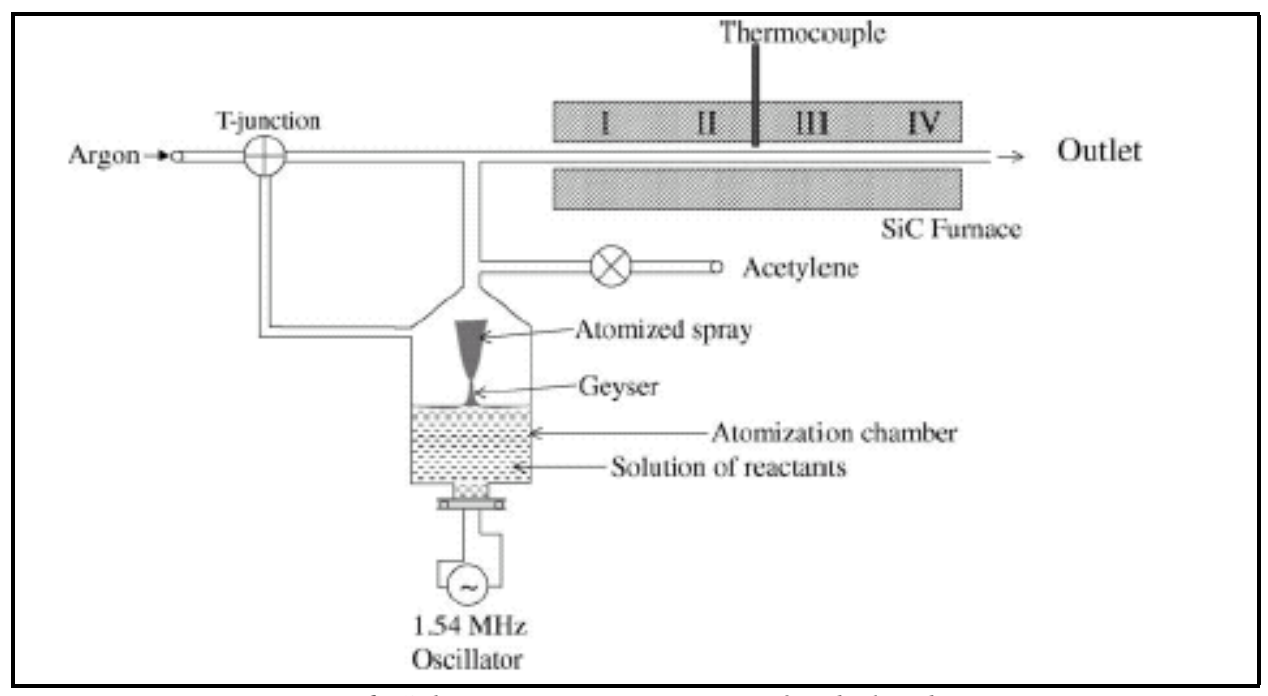

Figure 6: Schematic representation of Nebulized spray pyrolysis technique for synthesis of CNT

\section{Properties of CNT}

\section{Electrical properties}

The Unique Electrical Properties of carbon nanotubes are to a large extent derived ${ }^{29}$ from their 1-D character and the peculiar electronic structure of graphite. They have extremely low electrical resistance. Resistance occurs when an electron collides with some defect in the crystal structure of the material through which it is passing. The defect could be an impurity atom, a defect in the crystal structure, or an atom vibrating 
about its position in the crystal. Such collisions deflect the electron from its path. But the electrons inside a carbon nanotube are not so easily scattered. Because of their very small diameter and huge ratio of length to diameter-a ratio that can be up in the millions or even higher. In a 3-D conductor, electrons have plenty of opportunity to scatter, since they can do so at any angle. Any scattering gives rise to electrical resistance. In a 1-D conductor, however, electrons can travel only forward or backward. Under these circumstances, only backscattering (the change in electron motion from forward to backward) can lead to electrical resistance. But backscattering requires very strong collisions and is thus less likely to happen. So the electrons have fewer possibilities to scatter. This reduced scattering gives carbon nanotubes their very low resistance. In addition, they can carry the highest ${ }^{30}$ current density of any known material, measured ${ }^{31}$ as high as $10^{9} \mathrm{~A} / \mathrm{cm}^{2}$. One use for nanotubes that has already been developed is as extremely fine electron guns, which could be used as miniature cathode ray tubes (CRTs) in thin high-brightness low-energy low-weight displays. This type of display would consist of a group of many tiny CRTs, each providing the electrons to hit the phosphor of one pixel, instead of having one giant CRT whose electrons are aimed using electric and magnetic fields. These displays are known as Field Emission Displays (FEDs). A nanotube formed by joining nanotubes of two different diameters end to end can act as a diode, suggesting the possibility of constructing electronic computer circuits entirely out of nanotubes. Nanotubes have been shown to be superconducting at low temperatures.

\section{Mechanical properties}

The carbon nanotubes are expected to have high stiffness and axial strength as a result of the carbon-carbon $\mathrm{sp}^{2}$ bonding ${ }^{32}$. The practical application of the nanotubes requires the study of the elastic response, the inelastic behavior and buckling, yield strength and fracture. Efforts have been applied to the experimental ${ }^{33-36}$ and theoretical investigation of these properties. Nanotubes are the stiffest known fiber, with a measured Young's modulus $^{37}$ of $1.4 \mathrm{TPa}$. They have an expected elongation to failure of $20-30 \%$, which combined with the stiffness, projects to a tensile strength well above $100 \mathrm{GPa}$ (possibly higher), by far the highest known. For comparison, the Young's modulus ${ }^{38}$ of high-strength steel is around $200 \mathrm{GPa}$, and its tensile strength is 1-2 GPa.

\section{Thermal Properties}

Prior to CNT, diamond was the best thermal conductor. CNT have now been shown to have a thermal conductivity at least twice that of diamond ${ }^{39}$. CNT have the unique property of feeling cold to the touch, like metal, on the sides with the tube ends exposed, but similar to wood on the other sides. The specific heat and thermal conductivity of carbon nanotube systems are determined primarily by phonons. The measurements yield linear specific heat and thermal conductivity above $1 \mathrm{~K}$ and below room temperature ${ }^{40-45}$ while a $\mathrm{T}^{0.62}$ behavior of the specific heat was observed below $1 \mathrm{~K}$. The linear temperature dependence can be explained with the linear k-vector dependence of the frequency of the longitudinal and twist acoustic phonons ${ }^{46}$. The specific behavior 
of the specific heat below $1 \mathrm{~K}$ can be attributed to the transverse acoustic phonons with quadratic $\mathrm{k}$ dependence ${ }^{47-50}$. The measurements of the thermoelectric power (TEP) of nanotube systems give direct information for the type of carriers and conductivity mechanisms.

\section{CARBON NANOTUBES COMPOSITES}

\section{Polymer matrix composite}

Since the documented discovery of carbon nanotubes (CNT) in 1991 by Iijima $^{3}$ and the realization of their unique ${ }^{51}$ physical properties, including mechanical, thermal, and electrical, many investigators have endeavored to fabricate advanced CNT composite materials that exhibit one or more of these properties. For example, as conductive filler in polymers, CNT are quite effective compared to traditional carbon black microparticles, primarily due to their high aspect ratios. The electrical percolation threshold was recently reported at $0.0025 \mathrm{wt}$. \% CNT and conductivity at $2 \mathrm{~S} / \mathrm{m}$ at $1.0 \mathrm{wt} \% \mathrm{CNT}$ in epoxy matrices $^{52}$ (figure 7 ).

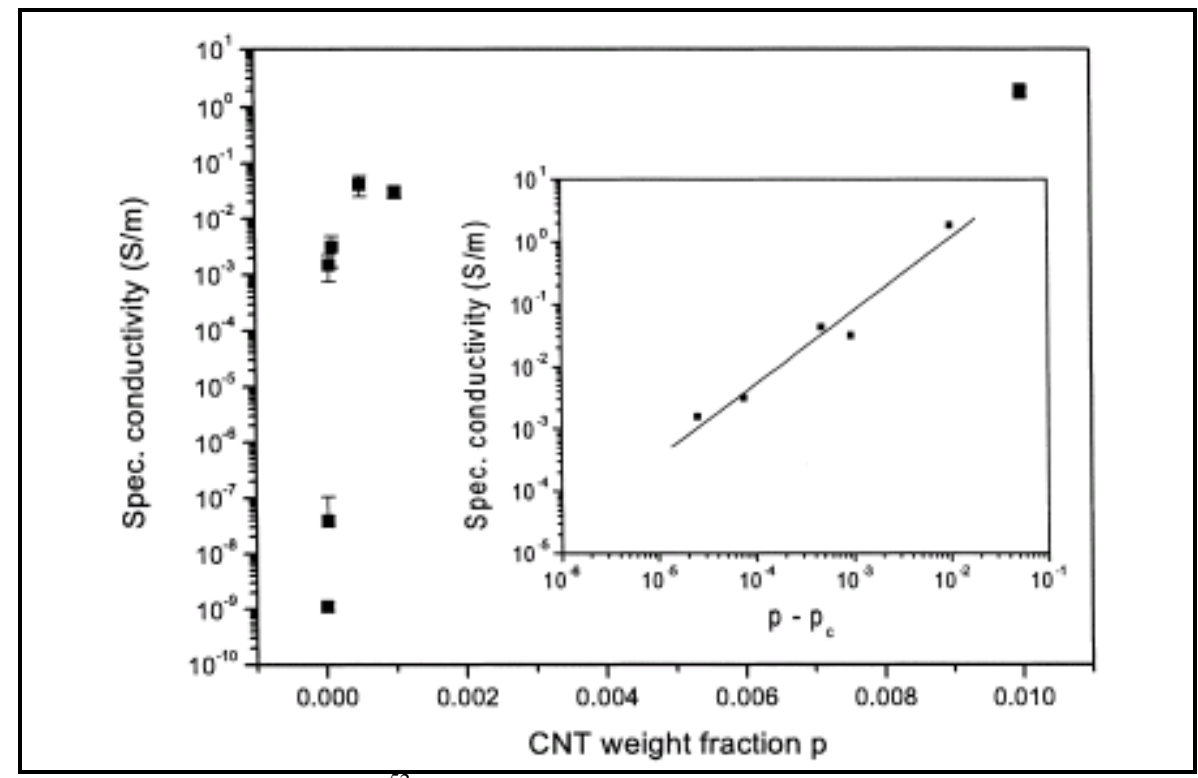

Figure 7: Semi- log plot ${ }^{52}$ of the specific composite conductivity as a function of carbon nanotube weight fraction $p$ : The insert shows a log-log plot of the conductivity as a function of $p-p_{c}$ with an exponent $t$ of 1.2

Similarly, CNT possess one of the highest thermal conductivities known ${ }^{53}$, which suggests their use in composites for thermal management. The CNT can be thought of as the ultimate carbon fiber with break strengths reported as high as $200 \mathrm{GPa}$, and elastic moduli in the $1 \mathrm{TPa}$ range ${ }^{54-55}$. This, coupled with approximately 500 times more surface area per gram (based on equivalent volume fraction of typical carbon fiber) and aspect ratios of around 103, has spurred a great deal of interest in using CNT as a reinforcing phase for polymer matrices. Increasing attention is being focused on the CNT surface, 
namely the interface between the CNT and surrounding polymer matrix. From micromechanics, it is through shear stress build-up at this interface that stress is transferred from the matrix to the CNT. Numerous researchers have attributed lower-than-predicted CNT-polymer composite properties to a lack of interfacial bonding ${ }^{56,57}$. If one considers the surface of a CNT, essentially an exposed graphene sheet, it is not surprising that interfacial interaction is a concern. It is the weak inter-planar interaction of graphite that provides its solid lubricant quality, and resistance to matrix adhesion. This is exaggerated by the chemically inert nature of graphene structures. Significant toughening of polymer matrices through the incorporation of CNT has been reported ${ }^{58}$. A loading of $1 \mathrm{wt} \%$ MWNT, randomly distributed in an ultra-high molecular weight polyethylene was reported to increase the strain energy density by $\sim 150 \%$ and increase the ductility by $\sim 140 \%$. Secondary crystallites, which nucleated from the MWNT, were attributed a higher mobility and hence the increase in strain energy ${ }^{59}$. A similar effect was found in aligned MWNT/polyacrylonitrile. Fibers containing 1.8 vol. \% MWNT with an approximately $80 \%$ increase in energy to yield and energy to break ${ }^{56}$. A process of spinning $60 \mathrm{wt} \%$ SWNT/polyvinylalcohol fibers with pre-drawn energy absorbing capacity nearly 3.5 times spider silk (165 J/g) was reported ${ }^{58}$. Slippage between SWNT bundles was suggested as the mechanism responsible for the enhancement in the toughness. The addition of $1 \mathrm{wt} \%$ MWNT to isotactic polypropylene (iPP) was shown to affect crystal nucleation from differential scanning calorimetry and X-ray diffraction measurements ${ }^{60}$. Compared with neat iPP, there was an increase in crystallization rate for the composite material with evidence of fibrillar crystal growth rather than spherulite growth. These modifications in the morphology of a polymer matrix combined with the energy required for CNT debonding and pull-out suggest CNT may augment the energy absorption or toughness characteristics of the composite. A twofold increase in the tension-tension fatigue strength for an aligned SWNT/epoxy composite was found in comparison to typical carbon fiber/epoxy composites. Embedded $\mathrm{CNT}^{61}$ may effectively prolong the formation of and/or bridge micro cracking/ crazing that can propagate and lead to fatigue failure. CNT reinforced polymer composites are seen as a potentially fruitful area for new, tougher or fatigue resistant materials. Further investigations into the toughness and fatigue properties of these composites are needed to understand the reinforcing mechanism at work.

The influence of carbon nanotube (CNT) contents on electrical and rheological properties of CNT-reinforced polypropylene (PP) composites was studied. As a result, the volume resistivity of the composites was decreased with ${ }^{62}$ increasing the CNT content and the electrical percolation threshold was formed between 1 and 2 wt\% CNT, which were caused by the formation of conductive chains in the composites (figure 8). And the viscosity of the composites was increased with the addition of CNT, which was accompanied by an increase in elastic melt properties (figure 9). This could be explained by the higher aspect ratio of the CNT. And the composites containing more than $2 \mathrm{wt} \%$ CNT exhibited non-Newtonian curves at low frequency.

Polyimide/carbon nanotube (PI/CNT) nanocomposites ${ }^{63}$ with different proportions of CNT were fabricated by in situ process. The bending strength and microhardness of the PI/CNT nanocomposites were measured. The friction and wear 
behavior of the nanocomposites was evaluated on an M-2000 friction and wear tester. The results showed that the bending strength and microhardness of the PI/CNT nanocomposites increased with increasing CNT content and reached stable values at a certain content of CNT. CNT could effectively enhance the friction-reduction and antiwear capacity of the nanocomposite because it increased the load capacity and mechanical strength of the $\mathrm{CNT} / \mathrm{PI}$. The variables such as applied load and sliding speed had a significant influence on

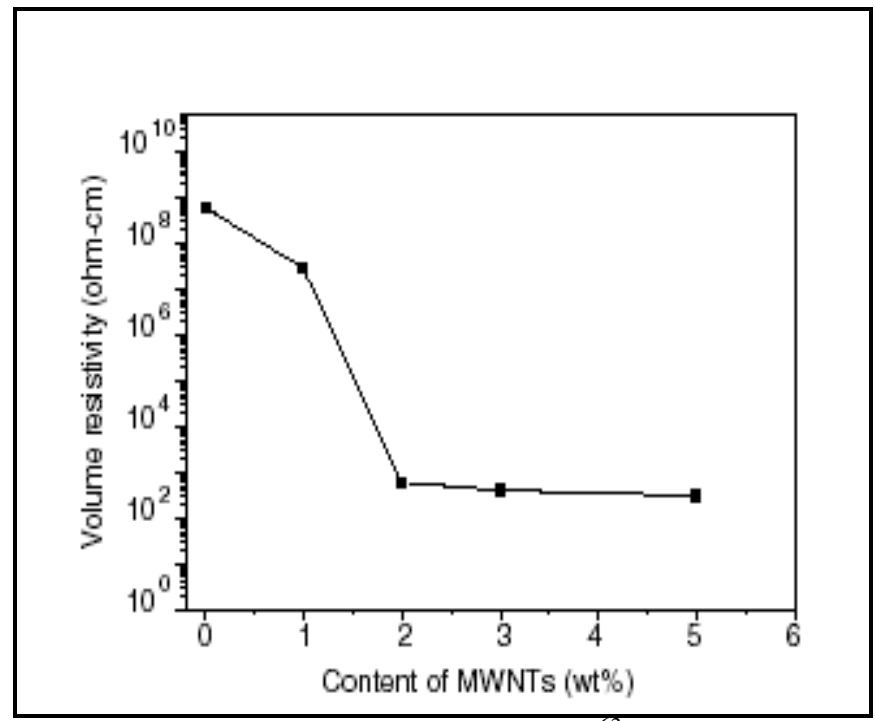

Figure 8: Electrical volume resistivity ${ }^{62}$ of MWNT/PP composites as a function of nanotube content.

friction and wear performance.

Composites of high molecular weight polyaniline (PANI) ${ }^{64}$ and various weight percentages of singlewalled carbon nanotubes (SWNT) were fabricated using solution processing. Electrical characteristics of metal-semiconductor (MS) devices fabricated from the PANI/SWNT composites were studied. Currentvoltage (I-V) characteristics of these devices indicate a significant increase in current with an increase in carbon nanotube concentration in the composite.

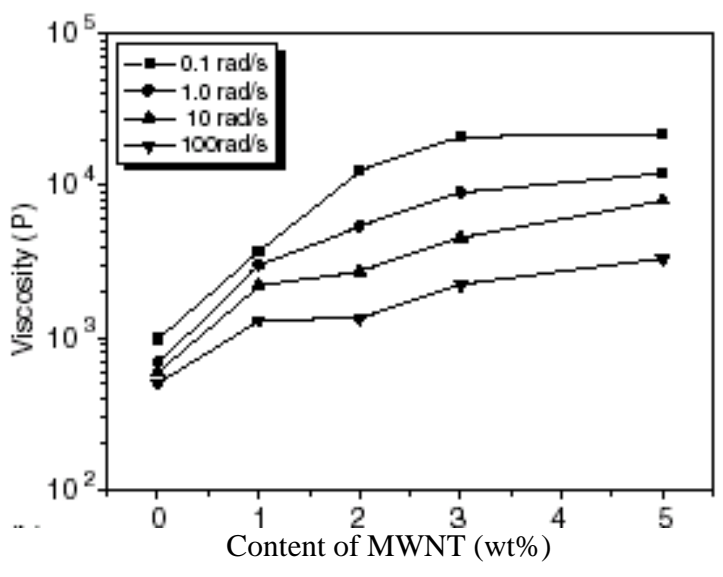

Figure 9: Viscosity of MWNT/PP composites ${ }^{62}$ measured at $170^{\circ} \mathrm{C}$

Emilie et al. ${ }^{65}$ found that the tensile modulus and yield strength increased with the addition of SWNT loading in a polyimide SWNT composite. The increase was much higher than that observed for film samples (which were cast without preferred SWCNT orientation), but much less than what was expected from an oriented discontinuous fiber reinforced polymer composite. This low level of improvement was likely due to inefficient and incomplete dispersion. With the aid of improved dispersion, significant reinforcing effects of the aligned fibers on the mechanical properties are anticipated. Improvements in mechanical reinforcement may also be realized with a matrix designed to promote uniform dispersion by capitalizing on physical interaction with SWCNT inclusions to improve the nanotube/matrix interface so as to maximize load transfer across the interface. 
In a study by Pötschke et al. a masterbatch of PCMWNT $^{66}$ (15 wt\%) was diluted with different amounts of PC in a small scale conical twin screw extruder (DACA Micro Compounder) to obtain different compositions of MWNT. In this system, electrical measurements indicated percolation of MWNT between 1.0 and $1.5 \mathrm{wt}$ $\%$ ( figure10).

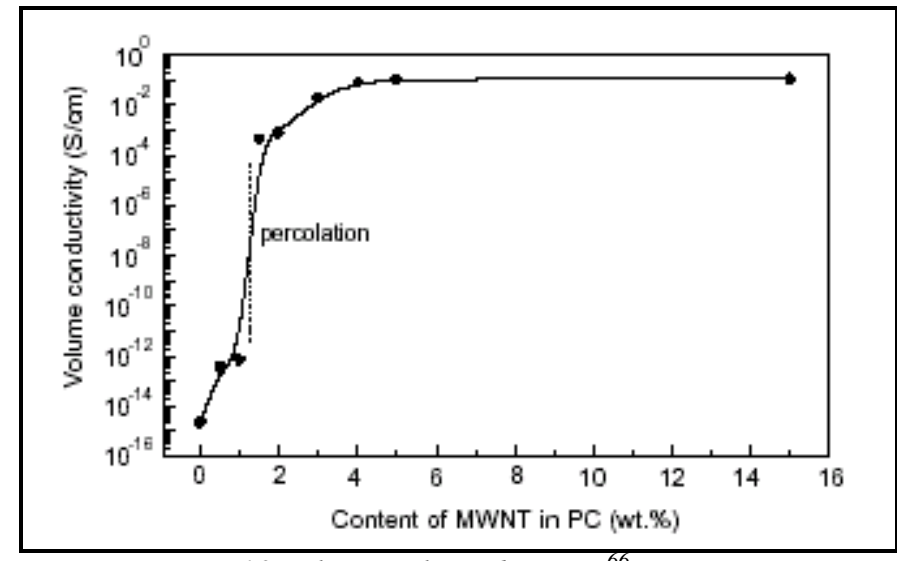

Figure 10: Electrical conductivity ${ }^{66}$ vs. MWNT contents for PC-MWNT composites.

A novel

polyacrylamide-carbon nanotubes ${ }^{67}$ (PAM-CNT) copolymer has been prepared by ultraviolet radiation initiated polymerization. The PAM-CNT copolymer was characterized by the instruments of Fourier transform infrared spectroscopy, UV-vis absorbance spectra, fluorescence spectra and transmission electron microscope. The morphology and microtribological properties of PAM-CNT thin films on mica were investigated by atomic force microscopy/friction force microscopy (AFM/FFM). The friction of the films was stable with the change of applied load and the friction coefficient decreased significantly as the CNT addition. The results show that the rigid rod-like CNT in polymer would enhance load-bearing and anti-wear properties of the thin films.

SMA encapsulated $\mathrm{SWNT}^{68}$ are melt mixed with PA12 matrix in a conical twinscrew extruder. The process of encapsulation by SMA copolymer leads to a finer dispersion of SWNT and enhanced interfacial adhesion between PA12 and SMA modified SWNT. This leads to enhanced mechanical properties, which is manifested by tensile and dynamic mechanical properties. Formation of network structure (figure 11) has been identified in

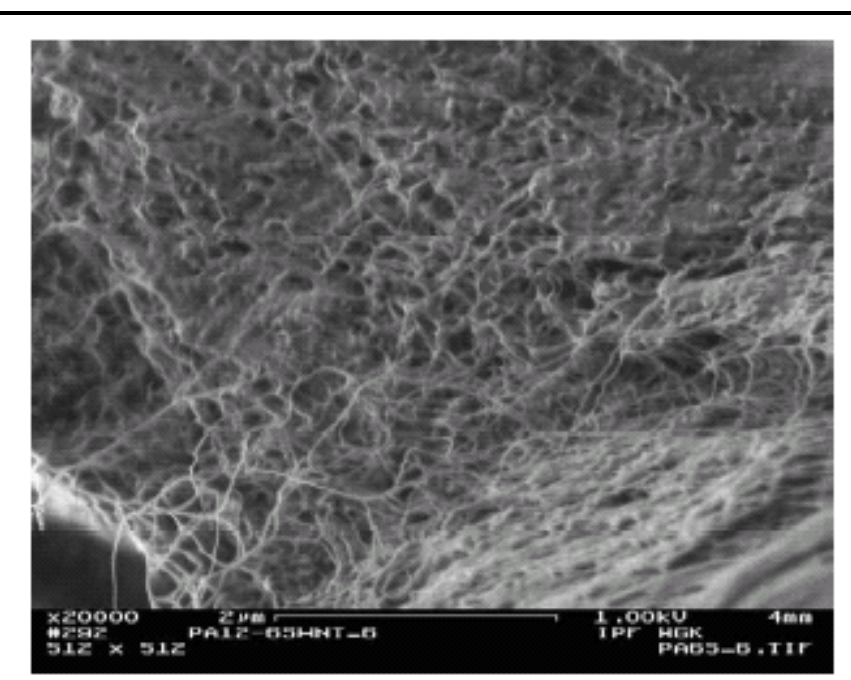

Figure 11: SEM of the composite ${ }^{68}$ PA12+6 wt\% SWNT after tensile testing, area near fracture surface. unmodified SWNT composites by electrical conductivity measurements and morphological investigations by scanning electron microscopy. 
Zou et al. showed that for the dispersion of MWNT in a polymer ${ }^{69}$ matrix by screw extruder, there is a critical MWNT concentration of $1.0 \mathrm{wt} \%$ where a fine network of filler is formed; therefore the composites possess improved mechanical properties.

Shuying et al. found from DSC analysis that the introduction of $\mathrm{SWNT}^{70}$ increases the glass transition temperature of the composites and low concentration of SWNT act as nucleating agents to the crystallization of ABS as small melting peaks were observed at $0.5 \mathrm{wt} \%$ and $1 \mathrm{wt} \%$ of SWNT.

\section{Significant improvements in the mechanical properties $^{71}$ of the epoxy/SWNT nanocomposites were illustrated by a $50.8 \%$ increase in the storage modulus by Liao et al. (figure 12).}

The significant
improvements of
nanotube dispersion
and mechanical
performance were
attributed to the
combined use of tip sonication and acetone as dispersion aids during sample processing.

Nanocomposites consisting of double-wall carbon nanotubes ${ }^{72}$ (DWCNT) and an epoxy matrix were produced by a standard calandering technique. A very good dispersion of both DWCNT and carbon black (CB) in an epoxy resin could be observed. The investigation of the (fracture-) mechanical properties resulted in an increase of strength, Young's modulus and strain to failure at a nanotube content of only $0.1 \mathrm{wt} \%$. The correlation of the experimentally obtained Young's moduli showed a good agreement with a modified Halpin-Tsai theory.

Poly (methyl methacrylate) (PMMA) nanocomposites have been processed ${ }^{73}$ by melt blending. The amount of nano fibers used was 5 and $10 \mathrm{wt} \%$, respectively. The PMMA/CNF composites were processed into $4 \mathrm{~mm}$ diameter rods and $60 \mathrm{~mm}$ diameter fibers using small-scale melt spinning equipment. At $5 \mathrm{wt} \% \mathrm{CNF}$, composite rods as well as fibers show over $50 \%$ improvement in axial tensile modulus as compared to the control PMMA rod and fibers, respectively. The reinforcement efficiency decreased at 10 wt\% CNF. The PMMA/CNF nanocomposites fibers also show enhanced thermal 
stability, significantly reduced shrinkage and enhanced modulus retention with temperature, as well as improved compressive strength.

Polystyrene nanocomposites ${ }^{74}$ with functionalized single-walled carbon nanotubes (SWNT), prepared by the in-situ generation and reaction of organic diazonium compounds, were characterized using melt-state linear dynamic viscoelastic measurements. These were contrasted to the properties of polystyrene composites prepared with unfunctionalized SWNT at similar loadings. The functionalized nanocomposites demonstrated a percolated SWNT network structure at concentrations of 1 vol \% SWNT, while the unfunctionalized SWNT-based composites at twice the loading of SWNT exhibited viscoelastic behavior comparable to that of the unfilled polymer. This formation of the SWNT network structure for the functionalized SWNT-based composites is because of the improved compatibility between the SWNT and the polymer matrix and the resulting better dispersion of the SWNT.

\section{Ceramic matrix}

Several experiments have recently confirmed $^{75}$ the theoretically predicted outstanding mechanical properties of carbon nanotubes (CNT). Consequently, CNT emerge as potentially attractive materials as reinforcing elements in ceramic matrix composites. Massive $\mathrm{CNT}-\mathrm{Fe}-\mathrm{Al}{ }_{2} \mathrm{O}_{3}$ composites have been prepared by hot pressing. Observations show that the CNT bundles remain present in the composites, but in a smaller quantity than in the starting powder. The improvement of the composite microstructure, the change in the nature of the matrix and attempts to align the CNT are works actually in progress. CNT-ceramic composites become attractive materials ${ }^{76}$ not only for their enhanced mechanical properties, but also for the possibility to tailor the electrical conductivity through the CNT content. A family of SWNT-MgAl ${ }_{2} \mathrm{O}_{4}$ massive composites has been prepared, in a wide range of composition $(0.23-24.5$ vol\% CNT) and with a very homogeneous distribution of the SWNT between the matrix grains owing to the in situ CCVD synthesis route. A jump of the DC electrical conductivity has been measured. Up to 11 vol\% CNT, the electrical conductivity is well fitted by the scaling law of the percolation theory. CNT-metal-oxide composites ${ }^{77}$ have been extruded at high temperatures. For temperatures up to $1500 \mathrm{C}$, some of the CNT remain undamaged neither by the high temperature nor by the extreme shear stresses. Moreover, the superplastic forming is made easier by the CNTs, which inhibit the matrix grain growth and also acts as a lubricating agent. It has been shown for the first time that it is possible to align CNTs in ceramic-matrix nanocomposites. CNT-Fe- $\mathrm{Al}_{2} \mathrm{O}_{3}$ composites have ${ }^{78}$ been prepared by hot-pressing composite powders where the quantity of CNT has greatly been increased in comparison with previous research.

\section{REFERENCES}

1. N .Valentin, Popov, Mat Sci and Engg R 43 (2004) 61.

2. H.W. Kroto, J.R. Heath, S.C. O’Brien, R.F. Curl, R.E. Smalley, Nature 318 (1985) 162. 
3. S. Iijima, Nature (London) 354 (1991) 56.

4. S. Iijima, T. Ichihashi, Nature (London) 363 (1993) 603.

5. D.S. Bethune, C.H. Kiang, M.S. de Vries, G. Gorman, R. Savoy, J. Vazquez, R. Beyers, Nature (London) 363 (1993) 605.

6. Ray H. Baughman, Anvar A. Zakhidov, Walt A. de Heer, Science 297(2002).

7. A. Thess, R. Lee, P. Nikolaev, H. Dai, P. Petit, J. Robert, C. Xu, Y.H. Lee, S.G. Kim, A.G. Rinzler, D.T. Colbert,G.E. Scuseria, D. Tomane’k, J.E. Fischer, R.E. Smalley, Science 273(1996) 483.

8. M.J. Yacaman, M.M. Yoshida, L. Rendon, J.G. Santiesteban, Appl. Phys. Lett. 62 (1993) 202.

9. $\quad$ T.W. Ebbesen, P.M. Ajayan, Nature (London) 358 (1992) 220.

10. C. Journet, W.K. Maser, P. Bernier, A. Loiseau, M. Lamy de la Chapelle, S. Lefrant, P. $\quad$ Deniard, R. Lee, and J.E. Fischer, Nature (London) 388 (1997) 756.

11. J. Liu, A.G. Rinzler, H. Dai, J.H. Hafner, R.K. Bradley, P.J. Boul, A. Lu, T. Iverson, K. Shelimov, C.B. Huffman, F. Rodriguex-Macia, D.T. Colbert, R.E. Smalley, Science 280 (1998) 1253.

12. A.G. Mamalis, L.O.G. Vogtländer, A. Markopoulos, Precision Engineering 28 (2004) 16.

13. W.Z. Li, S.S. Xie, L.X. Qian, B.H. Chang, B.S. Zou, W.Y. Zhou, Science 274(1996)1701

14. S.Xie, W.Li, Z.Pan, B.Chang, L.Sun , Mater Sci Eng A 286(2000)11.

15. S.Fan, W.Liang, H.Dang, N.Franklin, T.Tombler, M.Chapline, Phys E: LowDimensional Syst Nanostructures 8(2) (2000)179.

16. X.Y.Zhang , L.D.Zhang, G.H.Li, L.X.Zhao . Mater Sci Eng A 308(2001) 9

17. Z.K.Tang, L.Zhang, N.Wang, X.X.Zhang, G.H.Wen, G.D.Li, Science 292(2001) 2462

18. H.Ago, S.Obshima, K.Uchida, M.Yumura, J Phys Chem B 105(2001) 10453

19. Z.F.Ren, Z.P.Huang, J.W.Xu, J.H.Wang, P.Bush, M.P.Siegal, Science 282(1998) 1105

20. X.Wang, Z.Hu, X.Chen, Y.Chen, Scripta Mater 44(2001) 1567.

21. Kirsten Edgar, L.John, Spencer Current Applied Physics 4 (2004) 121

22. Luciano Andrey Montoro, Renata C.Z. Lofrano, Jose Maurý'cio Rosolen (article in press) Carbon (2004)

23. J.Livage, Solid State Ionics 86(1996) 935.

24 Jieshan Qiu, Yongfeng Li, Yunpeng Wang, Wen Li, Fuel Processing Technology 85 (2004) 1663.

25. Mingwang Shao, Debao Wang, Guihua Yu, Bing Hu, Weichao Yu, Yitai Qian Carbon 42 (2004) 183.

26. Ya-Li Li, Ian A. Kinloch, Milo S.P. Sha.er, Junfeng Geng, Brian Johnson, Alan H. Windle, Chemical Physics Letters 384 (2004) 98.

27. Ming-wei Li, Zheng $\mathrm{Hu}, \mathrm{Xi}$-zhang Wang, Qiang $\mathrm{Wu}, \mathrm{Yi}$ Chen, Yi-Ling Tian, Diamond and Related Materials 13 (2004) 111.

28 C.N.R. Rao, A. Govindaraj, Gautam Gundiah, S.R.C. Vivekchand, Chemical Engineering Science (article in press) 2004.

29. Ph. Avouris, J. Appenzeller, R. Martel, and S. Wind, Proceedings of the IEEE 91(2003) 1772. 
30. Sander J. Tans, Alwin R. M. Verschueren, Cees Dekker, Nature 393 (1998) 49.

31. B.Q. Wei, Appl. Phys. Lett. 79 (2001)1172 .

32. D.H. Robertson, D.W. Brenner, J.W. Mintmire, Phys. Rev. B 45 (1992)12592.

33. M.M.J. Treacy, T.W. Ebbesen, and J.M. Gilson, Nature (London) 381 (1996) 678.

34. A. Krishnan, E. Dujardin, T.W. Ebbesen, P.N. Yianilos, M.M.J. Treacy, Phys. Rev. B 58 (1998) 14013.

35. E.W. Wong, P.E. Sheehan, C.M. Lieber, Science 277 (1997) 1971.

36. M.F. Yu, O. Lourie, M.J. Dyer, K. Moloni, T.F. Kelly, R.S. Ruoff, Science 287 (2000) 637.

37. M.F. Yu , Phys. Rev. Lett. 84(2000) 5552

38. B.I. Yakobson, C.J. Brabec, J. Bernholc, Phys. Rev. Lett. 76 (1996) 2511.

39. J.Hone, Applied Physics, 273 (2001).

40. W. Yi, L. Lu, D.L. Zhang, Z.W. Pan, S.S. Xie, Phys. Rev. B 59 (1999) 9015.

41. A. Mizel, L.X. Benedict, M.L. Cohen, S.G. Louie, A. Zettl, N.K. Budraa, W.P. Beyermann, Phys. Rev. B 60 (1999) 3264.

42. J. Hone, B. Batlogg, Z. Benes, A.T. Johnson, J.E. Fischer, Science 289 (2000) 1730.

43. J.C. Lasjaunias, K. Biljakoviæ, Z. Benes, J.E. Fischer, P. Monceau, Phys. Rev. B 65 (2002) 113409.

44. J. Hone, M. Whitney, C. Piskoti, A. Zettl, Phys. Rev. B 59 (1999) 2514.

45. P. Kim, L. Shi, A. Majumdar, P.L. McEuen, Phys. Rev. Lett. 87 (2001) 215502.

46. L. X Benedict, S. G Louie, M.L. Cohen, Solid State Comm. 100 (1996) 177.

47. N. Popov, Phys. Rev. B 66 (2002) 153408.

48. J. Hone, I. Ellwood, M. Muno, A. Mizal, M.L. Cohen, A. Zettl, A.G. Rinzler, R.E. Smalley, Phys. Rev. Lett. 80 (1998) 1042.

49. M. Tian, F. Li, L. Chen, Z. Mao, Phys. Rev. B 58 (1998) 1166.

50. L. Grigorian, G.U. Sumanasekera, A.L. Loper, S.L. Fang, J.L. Allen, P.C. Eklund, Phys. Rev. B 60 (1999) 11309.

51. R. Andrews, M.C. Weisenberger, Current Opinion in Solid State and Materials Science 8 (2004) 31.

52. J.K.W.Sandler, J.E.Kirk, I.A.Kinloch, M.S.P.Sha, A.H.Windle, Polymer 44 (2003) 5893

53. P. Kim, L. Shi, A.Majumdar, P.L.McEuen, Phys Rev Lett 87 (2001) 2155021

54. K.T.Lau, D.Hui, Composites Part B 33(2002)263.

55. B.G.Demczyk, Y.M.Wang, J.Cumings, M.Hetman, W.Han, A.Zettl, Mater Sci Eng A 334(2002)173

56. M.C.Weisenberger, E.A.Grulke, D.Jacques, T.Rantell, R.Andrews, Nanotech 3(2003)

57. C.W.Nan, Z.Shi , Y.Lin ,Chem Phys Lett 375(2003) 666

58. A.B.Dalton, S.Collins, E.Munoz, J.M.Razal, V.H.Ebron, J.P.Ferraris, Nature 423(2003)703,

59. S.L.Ruan, P.Gao, X.G.Yang, T.X.Yu. Polymer 44 (2003) 5643.

60. E.Assouline, A.Lustiger, A.H.Barber, C.A.Cooper, E.Klein, E.Wachtel, Polym Sci Part B 41(2003) 520.

61. Y.Ren, F.Li, H.M.Cheng, K.Liao, Carbon 41(2003) 2159.

62 Min-Kang Seo, Soo-Jin Park, Chemical Physics Letters 395 (2004) 44.

63. Hui Cai, Fengyuan Yan, Qunji Xue,Materials Science and Engineering A 364 (2004) 94. 
64. P.C. Ramamurthy, A.M. Malshe, W.R. Harrell, R.V. Gregory, K. McGuire, A.M. Rao Solid-State Electronics 48 (2004) 2019.

65. Emilie J. Siochia, Dennis C. Working, Cheol Park, Peter T. Lilleheia, Jason H. Rouse, Crystal C. Topping, Arup R. Bhattacharyya, Satish Kumar,Composites: Part B 35 (2004) 439

66. Petra Pötschke, A.R.Bhattacharyya, Andreas Janke, European Polymer Journal 40 (2004) 137.

67. Xuefeng Lia, Wenchao Guana, Haibiao Yanb, Lan Huang, Materials Chemistry and Physics 88 (2004) 53

68. A.R. Bhattacharyya, Petra Pötschke, Mahmoud Abdel-Goad, Dieter Fischer Chemical Physics Letters 392 (2004) 28

69. Yaobang Zou, Yongcheng Feng, Lu Wang, Xiaobo Liu, Carbon 42 (2004) 271

70. Shuying Yang, Jose Rafael Castilleja, E.V. Barrerab, Karen Lozanoa, Polymer Degradation and Stability 83 (2004) 383.

71. Yu-Hsuan Liao, Olivier Marietta-Tondin, Zhiyong Liang, Chuck Zhang, Ben Wang, Materials Science and Engineering A 385 (2004) 175.

72. F.H. Gojny, M.H.G. Wichmann, U. Kopke, B. Fiedler, K. Schulte, Composites Science and Technology 64 (2004) 2363.

73. Jijun Zeng, Bethany Saltysiak, W.S. Johnson, David A. Schiraldi, Satish Kumar, Composites: Part B 35 (2004) 173.

74. A.Cynthia, L.Jeffrey, Sivaram Arepalli, James M. Tour and R. Krishnamoorti, Macromolecules, 35 (2002) 8825

75. A. Peigney, Ch. Laurent, E. Flahaut, A. Rousset Ceramics International 26 (2000) 677-683

76. S. Rul, F. Lef_evre-schlick, E. Capria, Ch. Laurent, A. Peigney * Acta Materialia 52 (2004) 1061-1067

77. A. Peigney, E. Flahaut, Ch. Laurent, F. Chastel, A. Rousset Chemical Physics Letters 352 (2002) 20-25

78. E. Flahaut1, A. Peigney1, Ch. Laurent, Ch. Marlie`Re, F. Chastel And A. Rousset, Acta Mater. 48 (2000) 3803-3812 